\title{
Collisions on SHA-0 in One Hour
}

\author{
Stéphane Manuel ${ }^{1, \star}$ and Thomas Peyrin ${ }^{2,3,4, \star \star}$ \\ ${ }^{1}$ INRIA \\ stephane.manuel@inria.fr \\ 2 Orange Labs \\ thomas.peyrin@orange-ftgroup.com \\ ${ }^{3}$ AIST \\ ${ }^{4}$ Université de Versailles Saint-Quentin-en-Yvelines
}

\begin{abstract}
At Crypto 2007, Joux and Peyrin showed that the boomerang attack, a classical tool in block cipher cryptanalysis, can also be very useful when analyzing hash functions. They applied their new theoretical results to SHA-1 and provided new improvements for the cryptanalysis of this algorithm. In this paper, we concentrate on the case of SHA-0. First, we show that the previous perturbation vectors used in all known attacks are not optimal and we provide a new 2-block one. The problem of the possible existence of message modifications for this vector is tackled by the utilization of auxiliary differentials from the boomerang attack, relatively simple to use. Finally, we are able to produce the best collision attack against SHA-0 so far, with a measured complexity of $2^{33,6}$ hash function calls. Finding one collision for SHA-0 takes us approximatively one hour of computation on an average $\mathrm{PC}$.
\end{abstract}

Keywords: hash functions, SHA-0, boomerang attack.

\section{Introduction}

Cryptographic hash functions are an important tool in cryptography. Basically, a cryptographic hash function $H$ takes an input of variable size and returns a hash value of fixed length while satisfying the properties of preimage resistance, second preimage resistance, and collision resistance [11. For a secure hash function that gives an $n$-bit output, compromising these properties should require $2^{n}, 2^{n}$, and $2^{n / 2}$ operations respectively.

Usually, hash functions are built upon two components: a compression function and a domain extension algorithm. The former has the same security requirements that a hash function but takes fixed length inputs. The latter defines how to use the compression function in order to handle arbitrary length inputs. From the early beginning of hash functions in cryptography, designers relied on the pioneering work of Merkle and Damgård [817] concerning the domain extension

\footnotetext{
* The first author is supported in part by the french Agence Nationale de la Recherche under the project designation EDHASH, DPg/ANR-CI FA/VB 2007-010.

** The second author is supported by the Japan Society for Promotion of Science and the French RNRT SAPHIR project (http://www.crypto-hash.fr).
} 
algorithm. Given a collision resistant compression function, it became easy to build a collision resistant hash function. However, it has been recently shown that this iterative process presents flaws 9121315 and some new algorithms [14] with better security properties have been proposed. One can distinguish three different methods for compression function designs: block cipher based, related to a well studied hard problem and from scratch.

The most famous design principle for dedicated hash functions is indisputably the MD-SHA family, firstly introduced by R. Rivest with MD4 [24] in 1990 and its improved version MD5 23 in 1991. Two years after, the NIST publishes 19 a very similar hash function, SHA-0, that will be patched 20 in 1995 to give birth to SHA-1. This family is still very active, as NIST recently proposed [21] a 256-bit new version SHA-256 in order to anticipate the potential cryptanalysis results and also to increase its security with regard to the fast growth of the computation power. All those hash functions use the Merkle-Damgård extension domain and their compression function, even if considered conceived from scratch, is built upon a dedicated block cipher in Davies-Meyer mode: the output of the compression function is the output of the block cipher with a feed-forward of the chaining variable.

Dobbertin 10 provided the first cryptanalysis of a member of this family with a collision attack against MD4. Later, Chabaud-Joux [7] published the first theoretical collision attack against SHA-O and Biham-Chen [2] introduced the idea of neutral bits, which led to the computation of a real collision with four blocks of message [3. Then, a novel framework of collision attack, using modular difference and message modification techniques, surprised the cryptography community $26 / 27 / 28 / 29$. Those devastating attacks broke a lot of hash functions, such as MD4, MD5, SHA-0, SHA-1, RIPEMD or HAVAL-128. In the case of SHA-0 the overall complexity of the attack was $2^{39}$ message modification processes. Recently, Naito et al. 18] lower this complexity down to $2^{36}$ operations, but we argue in this paper that it is a theoretical complexity and not a measured one.

At Crypto 2007, Joux and Peyrin [14] published a generalization of neutral bits and message modification techniques and applied their results to SHA-1. The socalled boomerang attack was first devoted for block ciphers cryptanalysis 25. but their work showed that it can also be used in the hash functions setting. Used in parallel with the automated tool from De Cannière and Rechberger [5] that generates non-linear part of a differential path, this method turns out to be quite easy to use and handy for compression functions cryptanalysis.

This article presents a new attack against the collision resistance of SHA-0 requiring only $2^{33}$ hash computations and the theoretical analysis is confirmed by experimentation. First, we show that the previously used perturbation vector, originally found by Wang et al., is not optimal. We therefore introduce a new vector, allowing ourselves to use two iterations of the compression function. In order to compensate the loss of the known message modifications due to the perturbation vector change, we use the boomerang attack framework in order to accelerate the collision search. Finally, this work leads to the best collision 
attack against SHA-0 from now on, now requiring only one hour of computation on an average $\mathrm{PC}$.

We organized the paper as follows. In Section 2, we recall the previous attacks and cryptanalysis techniques for SHA-0. Then, in Section 3. we analyze the perturbation vector problem and give new ones that greatly improve the complexity of previous attacks. We then apply the boomerang technique as a speedup technique in Section 4 and provide the final attack along with its complexity analysis in Section 5. Finally, we draw conclusions in Section 6.

\section{Previous Collision Attacks on SHA-0}

\subsection{A Short Description of SHA-0}

SHA-0 [19], is a 160-bit dedicated hash function based on the design principle of MD4. It applies the Merkle-Damgård paradigm to a dedicated compression function. The input message is padded and split into $k$ 512-bit message blocks. At each iteration of the compression function $h$, a 160-bit chaining variable $H_{t}$ is updated using one message block $M_{t+1}$, i.e $H_{t+1}=h\left(H_{t}, M_{t+1}\right)$. The initial value $H_{0}$ (also called IV) is predefined and $H_{k}$ is the output of the hash function.

The SHA-O compression function is build upon the Davis-Meyer construction. It uses a function $E$ as a block cipher with $H_{t}$ for the message input and $M_{t+1}$ for the key input, a feed-forward is then needed in order to break the invertibility of the process:

$$
H_{t+1}=E\left(H_{t}, M_{t+1}\right) \boxplus H_{t},
$$

where $\boxplus$ denotes the addition modulo $2^{32} 32$-bit words by 32 -bit words. This function is composed of 80 steps (4 rounds of 20 steps), each processing a 32bit message word $W_{i}$ to update 532 -bit internal registers $(A, B, C, D, E)$. The feed-forward consists in adding modulo $2^{32}$ the initial state with the final state of each register. Since more message bits than available are utilized, a message expansion is therefore defined.

Message Expansion. First, the message block $M_{t}$ is split into 16 32-bit words $W_{0}, \ldots, W_{15}$. These 16 words are then expanded linearly, as follows:

$$
W_{i}=W_{i-16} \oplus W_{i-14} \oplus W_{i-8} \oplus W_{i-3} \text { for } 16 \leq i \leq 79 .
$$

State Update. First, the chaining variable $H_{t}$ is divided into 5 32-bit words to fill the 5 registers $\left(A_{0}, B_{0}, C_{0}, D_{0}, E_{0}\right)$. Then the following transformation is applied 80 times:

$$
S T E P_{i+1}:=\left\{\begin{array}{l}
A_{i+1}=\left(A_{i} \ll 5\right)+f_{i}\left(B_{i}, C_{i}, D_{i}\right)+E_{i}+K_{i}+W_{i}, \\
B_{i+1}=A_{i}, \\
C_{i+1}=B_{i} \gg 2, \\
D_{i+1}=C_{i}, \\
E_{i+1}=D_{i} .
\end{array}\right.
$$

where $K_{i}$ are predetermined constants and $f_{i}$ are boolean functions defined in Table 1] 
Feed-Forward. The sums modulo $2^{32}:\left(A_{0}+A_{80}\right),\left(B_{0}+B_{80}\right),\left(C_{0}+C_{80}\right)$, $\left(D_{0}+D_{80}\right),\left(E_{0}+E_{80}\right)$ are concatenated to form the chaining variable $H_{t+1}$.

Note that all updated registers but $A_{i+1}$ are just rotated copies, so we only need to consider the register $A$ at each step. Thus, we have:

$$
A_{i+1}=\left(A_{i} \ll 5\right)+f_{i}\left(A_{i-1}, A_{i-2} \gg 2, A_{i-3} \gg 2\right)+A_{i-4} \gg 2+K_{i}+W_{i} .
$$

\subsection{First Attacks on SHA-0}

The first published attack on SHA-0 has been proposed by Chabaud and Joux in 1998 [7. It focused on finding linear differential paths composed of interleaved 6-step local collisions, which have probability 1 to hold in a linearized version of SHA-0. However, in the standard version of SHA-0, a local collision only has a certain probability to hold. The overall probability of success of the attack is the product of the holding probability of each local collision.

The core of the differential path is represented by a perturbation vector which indicates where the 6 -step local collisions are initiated. The probability of success of the attack is then related to the number of local collisions appearing in the perturbation vector. In their paper, Chabaud and Joux have defined 3 necessary conditions on perturbation vectors in order to permit the differential path to end with a collision for the 80-step compression function. Such a perturbation vector should not have truncated local collisions, should not have two consecutive local collisions initiated in the first 16 th steps and should not start a local collision after step 74. Under these constraints they were able to find a perturbation vector (so-called L-characteristic) with a probability of success of $2^{68}$. The running complexity of their attack is decreased to $2^{61}$ by a careful implementation of the collision search. As the attacker has full control on the first 16 message blocks, those blocks are chosen such that the local collisions of those early steps hold with probability 1. See [7] for more details.

In 2004, Biham and Chen have improved the attack of Chabaud and Joux by introducing the neutral bit technique. The idea is to multiply the number of conformant message pairs up to a certain step $s$ (message pairs that verify the main differential path up to step $s$ ) for a cost almost null. This is done by looking for different sets of small modifications in the message words such that each set will have very low impact on the conformance of the message pair up to step s. Basically, the attacker can effectively start the collision search at a higher step than in a normal setting, and this improvement finally led to the

Table 1. Boolean functions and constants in SHA-0

\begin{tabular}{|c|c|c|c|}
\hline round & step $i$ & $f_{i}(B, C, D)$ & $K_{i}$ \\
\hline \hline 1 & $1 \leq i \leq 20$ & $f_{I F}=(B \wedge C) \oplus(\bar{B} \wedge D)$ & 0x5a827999 \\
\hline 2 & $21 \leq i \leq 40$ & $f_{X O R}=B \oplus C \oplus D$ & 0x6ed6eba1 \\
\hline 3 & $41 \leq i \leq 60$ & $f_{M A J}=(B \wedge C) \oplus(B \wedge D) \oplus(C \wedge D)$ & 0x8fabbcdc \\
\hline 4 & $61 \leq i \leq 80$ & $f_{X O R}=B \oplus C \oplus D$ & 0xca62c1d6 \\
\hline
\end{tabular}


computation of the first real collision for SHA-0 with four blocks of message [3] with an overall complexity of $2^{51}$ functions calls.

\subsection{The Wang Approach}

The attack on SHA-0 of Wang et al. is derived from the approach of Chabaud and Joux. The principle of this attack consists in relaxing two of the three conditions on the perturbation vectors defined by Chabaud and Joux, namely no truncated local collision allowed and no consecutive local collisions in the 16th first steps. Relaxing those conditions permits to search for better perturbation vectors, i.e. higher probability linear differential paths.

However, the main drawback of this approach is that non corrected perturbations inherited from truncated local collisions appear in the first steps. In order to offset these unwanted perturbations, they had to construct a non linear differential path (so-called NL-characteristic) which connects to the desired linear differential path. Said in other words, they kept the same linear differential mask on the message, but computed a new and much more complex differential mask on the registers for the early steps of SHA-0. A NL-characteristic presents also the advantage that consecutive local collisions in the early steps are no more a problem. Using modular subtraction as the differential, the carry effect (a property of the powers of 2 , i.e. $2^{j}=-2^{j}-2^{j+1} \ldots-2^{j+k-1}+2^{j+k}$ ) and by carefully controlling the non linearity of the round function IF, they succeeded to build their NL-characteristic by hand. A NL-characteristic holds only if specific conditions are verified step by step by the register values. In their paper, Wang et al. denoted these conditions as sufficient conditions. These sufficient conditions are described with respect to the register $A$ into one general form $A_{i, j}=v$ where $A_{i, j}$ denotes the value of bit $j$ of the register $A$ at the step $i$ and where $v$ is a bit value fixed to be 0 or 1 or a value that has been computed before step $i$.

The NL-characteristic found presents conditions on the initial value of the registers 1 . However, since the initial value is fixed, Wang et al. have build their collision with two blocks of message. The first block is needed in order to obtain a chaining variable verifying the conditions, inherited from the NL-characteristic, on the initial values of the register. This is detailed in Figure 1.

The attack of Wang et al. is thus divided into two phases. The first one is the pre-computation phase:

1. search for a higher probability L-characteristic by relaxing conditions on the perturbation vectors,

2. build a NL-characteristic which connects to the L-characteristic by offsetting unwanted perturbations,

3. find a first block of message from which the incoming chaining variable verifies the conditions inherited from the NL-characteristic.

${ }^{1}$ The conditions given by Wang et al. in their article are incomplete. In fact, two more conditions need to be verified 16[18. 


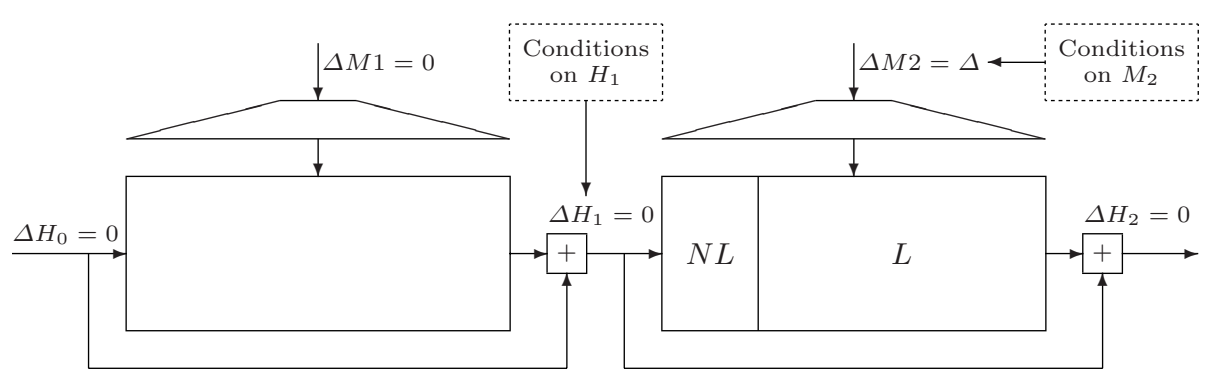

Fig. 1. Attack of Wang et al

The second phase is the collision search phase. It consists in searching for a second block of message for which the sufficient conditions on the register values are fulfilled for a maximum number of steps. In order to achieve that goal, they use both basic modification technique and advanced modification technique. The main idea of the former is simply to set $A_{i, j}$ to the correct bit by modifying the corresponding bit of the message word $W_{i-1}$. This is only possible for the first 16 steps, where the attacker has full control on the values $W_{i}$. The advanced modification technique are to be applied to steps 17 and higher, where the message words $W_{i}$ are generated by the message expansion. The idea is to modify the message words of previous steps in order to fulfill a condition in a given step. Wang et al. claimed in their article that using both basic modification and advanced modification techniques, they are able to fulfill all the sufficient conditions up to step 20. However very few details can be found on advanced modification technique in their article. Finally, their attack has a claimed complexity of $2^{39}$ SHA-0 operations.

Remark. Wang et al. optimized the choice of their perturbation vector taking into account their ability to fulfill the conditions up to step 20.

\subsection{Naito et al.}

Naito et al. recently proposed [18 a new advanced modification technique socalled submarine modification. Its purpose is to ensure that the sufficient conditions from steps 21 to 24 are fulfilled. The main idea of submarine modifications is to find modification characteristics which will permit to manipulate bit values of registers and message words after step 16. Each parallel characteristic is specifically built to satisfy one target condition. In order to construct such a characteristic, Naito et al. use two different approaches the cancel method and the transmission method. The former is based on the local collision principle. Whereas the transmission method combines the recurrence properties of the message expansion and of the step update transformation.

Those modification characteristics define new sets of conditions on register values and message words. The new conditions should not interfere with the already 
pre-computed sufficient conditions. Naito et al. detailed their submarine modifications up to step 17 (see [18] proof of Theorem 1). They remarked that the probability that one of these modifications can satisfy a target condition without affecting the other sufficient conditions is almost 1 . No detail is given about the impact of the submarine modifications after step 24.

The claimed complexity of the attack described in [18] is $2^{36}$ SHA-0 operations. This is a theoretical complexity that will be further discussed in section 5 . The given collision example is based on the same NL-characteristic and perturbation vector that Wang et al. used to produce their own collision. Taking into account that their submarine modifications permit to fulfill the sufficient conditions up to step 24, Naito et al. proposed a new perturbation vector which would therefore minimizes the complexity of the attack. However, in order to effectively build an attack based on the proposed vector, a new NL-characteristic and new submarine modifications should be found.

\section{A New Perturbation Vector}

In order to lower the complexity of a collision search on SHA-0, high probability L-characteristics are needed. In the previous attacks on SHA-0, the authors have proposed perturbation vectors which do not have local collisions starting after step 74. By relaxing this last condition, it may be possible to find better perturbation vectors. Note that those vectors do not seem to be eligible for a collision search, since they would lead to a near-collision (two compression function outputs with very few bits of difference) instead of a collision. However, this problem can be tackled by using the multi-block technique as in [3]: the attacker can take advantage of the feed-forward operation inherited from the Davis-Meyer construction used in the compression function of SHA-0. Said in other words, we allow ourselves to use several message blocks with differences, whereas the previous known attacks on SHA-0 only use one of such blocks of message. Thanks to the new automatic tool from De Cannière and Rechberger [5] that can generate NL-characteristics on SHA-1, computing non linear parts for SHA-0 is relatively easy. Indeed, SHA-1 and SHA-0 only differ on a rotation in the message expansion, which has no effect on the validity of this tool. Moreover, the ability to generate NL-characteristics reduce the multi-block problem to the use of only two blocks. More precisely, we start with a L-characteristic $L_{1}$ (defined by a new perturbation vector), and modified on the early steps by a generated NL-characteristic $N L_{1}$. We thus obtain a specific near-collision $\Delta H_{1}=+d$ after this first block. We then apply the same L-characteristic modified on the early steps by a second generated NL-characteristic $N L_{2}$, that takes in account the new incoming chaining variable $H_{1}$. Finally, before the feed-forward on this second block, we look for the opposite difference $\Delta E\left(H_{1}, m_{1}\right)=-d$ and the two differences cancel each over $\Delta H_{2}=0$. This is detailed in Figure 2.

Now that all the conditions on the perturbation vectors are relaxed, we need to define what are the criteria for good perturbation vectors. In order to fulfill the sufficient conditions inherited from $N L_{1}, N L_{2}$ and $L_{1}$, we will use basic 


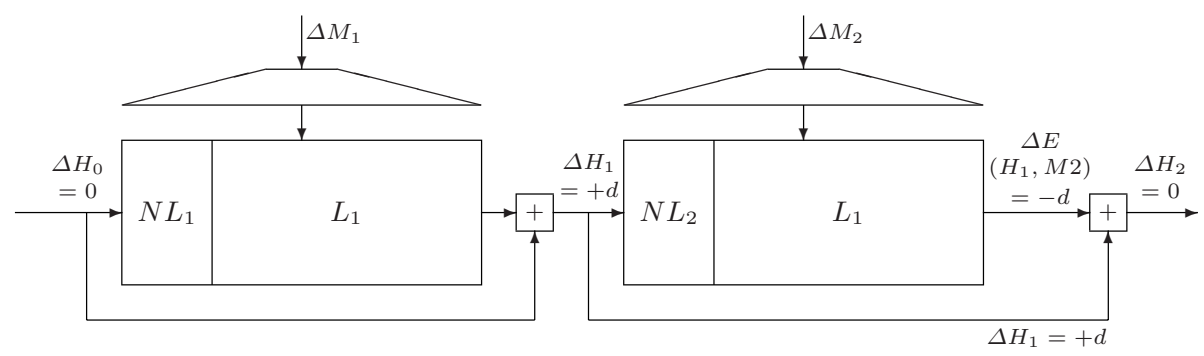

Fig. 2. Multi-block collision on SHA-0

Table 2. A new perturbation vector for SHA-0, along with the number of conditions at each steps (the conditions before step 16 have been removed since not involved in the complexity during the collision search)

\begin{tabular}{|c|c|}
\hline \multicolumn{2}{|r|}{ Steps 1 to 40} \\
\hline vector & 1111010110111000100010101000000000100100 \\
\hline$\sharp$ conditions & $\ldots$ \\
\hline
\end{tabular}

\begin{tabular}{|c|c|}
\hline & Steps 41 to 80 \\
\hline \hline vector & 00100000100001001011000000100001000010000 \\
\hline$\sharp$ conditions & 1110111010111101212211000010101010010100 \\
\hline
\end{tabular}

message modifications and boomerang techniques. In consequence, we focused our search on perturbation vectors that have the smallest number of sufficient conditions to fulfill in steps 17 to 80 . Namely, a characteristic $L_{1}$ for which the probability of success of the attack is maximized. We used the same approach of Chabaud and Joux in order to evaluate the probability of holding of each local collision involved. See [7] and particularly Section 2.2 (Tables 4 and 5) for detailed examples. There are a lot of perturbation vectors with an evaluated probability of success around $2^{-40}$ for the steps between 17 and 80 . However, this probability can be affected by the NL-characteristic. Thus, we build the NL-characteristics corresponding to each matching perturbation vector in order to compute the exact probability of success. This aspect of the search will be further detailed in the next section. The perturbation vector we chose, which has 42 conditions between step 17 and 80, is given in Table 2

\section{Boomerang Attacks for SHA-0}

Now that we found good perturbation vectors by relaxing certain conditions, a problem remains. Indeed, no message modification and no NL-characteristic are known for those vectors, and this makes the attack complexity drastically increase. This is the major drawback of Wang et al. collision attack on SHA-O and 
other hash functions: is not easily reusable and we are stuck with their perturbation vector. Hopefully, some work have been done recently in order to theorize Wang et al. major improvements. Recently, De Cannière and Rechberger 5] introduced an automated tool that generates non linear part of a differential path, thus resolving the NL-characteristic problem. Then, Joux and Peyrin [14] provided a framework that generalizes message modifications and neutral bits. Thanks to the so-called boomerang attack, they describe techniques that allows an attacker to easily use neutral bits or message modifications, given only a main perturbation vector. In fact, boomerang attacks and NL-characteristic automated search are exactly the two tools we need for our attack to be feasible. Finally, we replace the loss of the message modifications because of the new vector by the gain of the boomerang attack, which is a much more practical technique and fit for our constraints.

Boomerang attacks for hash functions can be seen as a generalization of collision search speed up techniques such as neutral bits or message modification. However, new possibilities were also suggested. In the usual setting, the attacker first sets the differential path and then tries to find neutral bits or message modifications if possible. In the explicit conditions approach from boomerang attacks, the attacker first set some constraints on the registers and the message words and then tries to find a differential path taking in account those constraints. One can see that for the latter part the NL-characteristic automated search tool becomes really handy. The constraints are set in order to provide very good message modifications or neutral bits that would not exist with a random differential path, or with very low probability. More generally, this can be seen as an auxiliary characteristic, different from the main one, but only fit for a few steps and this auxiliary characteristic can later be used as a neutral bit or a message modification, with very high probability (generally probability equal to 1) thanks to the preset constraints. Obviously the complexity of the collision search will decrease by adding as much auxiliary characteristics as possible.

Building an auxiliary path requires the same technique as for a main path, that is the local collisions. We refer to [14] for more details on this construction for SHA-1, since the technique is identically applicable to SHA-0. In our attack, we will consider two different types of auxiliary paths and we will use them as neutral bits (and not message modifications). Informally, we define the range of an auxiliary path to be the latest step where the uncontrolled differences from the auxiliary path (after the early steps) do not interfere in the main differential path. The first one, $A P_{1}$, will have very few constraints but the range will be low. On contrary, the second type, $A P_{2}$, will require a lot of constraints but the range will be much bigger. A trade-off among the two types needs to be considered in order not to have to many constraints forced (which would latter makes the NL-characteristic automated search tool fail) but also have a good set of auxiliary differential paths. More precisely, $A P_{1}$ and $A P_{2}$ are detailed in Figures 3 and 4 respectively. $A P_{1}$ is build upon only one local collision but the first uncontrolled difference appears at step 20. $A P_{2}$ is build upon three local collisions but the first uncontrolled difference appears at step 25. Note that, as remarked in the original paper from Joux 


\begin{tabular}{|c||c|c|}
\hline & $W_{0}$ to $W_{15}$ & $W_{16}$ to $W_{31}$ \\
\hline \hline perturbation mask & 0000001000000000 & \\
\hline \hline differences on $W^{j}$ & 0000001000000000 & 0000101101100111 \\
\hline differences on $W^{j+5}$ & 0000000100000000 & 0000010110110011 \\
\hline differences on $W^{j-2}$ & 0000000000010000 & 0001001000000010 \\
\hline
\end{tabular}

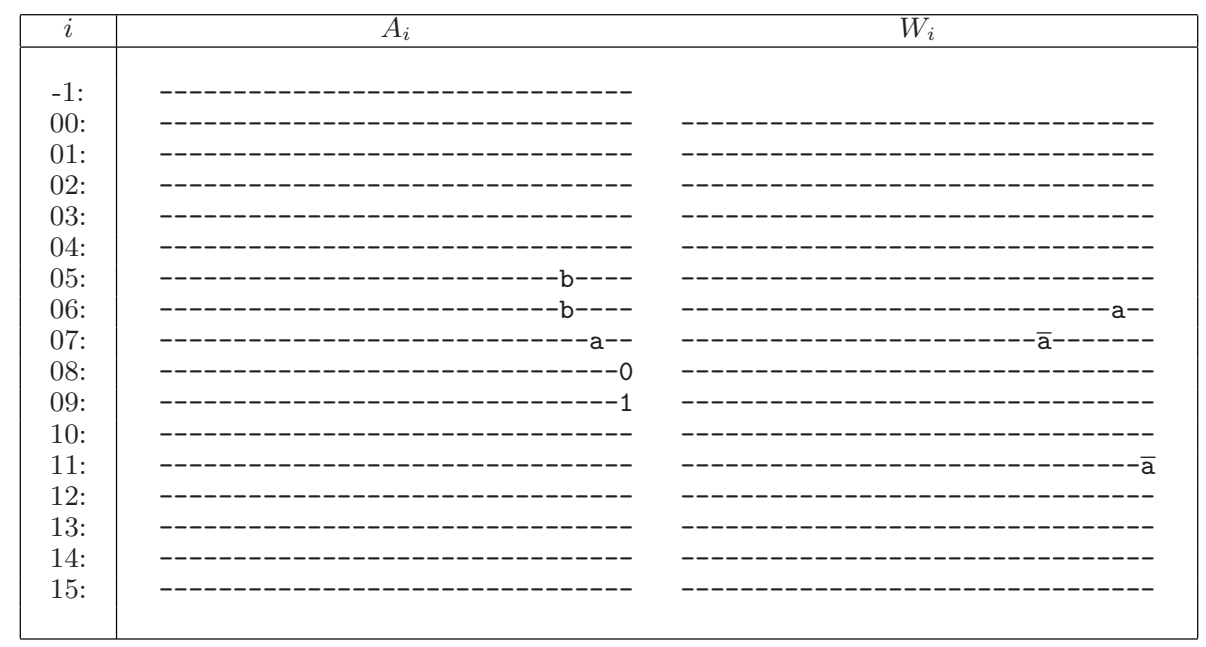

Fig. 3. Auxiliary differential path $A P_{1}$ used during the attack. The first table shows the 32 first steps of the perturbation vector (with the first uncontrolled difference on registers at step 20) and the second gives the constraints forced in order to have probability one local collisions in the early steps in the case where the auxiliary path is positioned at bit $j=2$. The MSB's are on the right and "-" stands for no constraint. The letters represent a bit value and its complement is denoted by an upper bar on the corresponding letter (see 14 for the notations).

and Peyrin, an auxiliary differential path used as a neutral bit with the first uncontrolled difference at step $s$ is a valid neutral bit for step $s+3$ with a very high probability (the uncontrolled difference must first propagate before disrupting the main differential path). Thus, in our attack, we will use $A P_{1}$ and $A P_{2}$ as neutral bits for steps 23 and 28 respectively; that is as soon as we will find a conformant message pair up to those step during the collision search, we will trigger the corresponding auxiliary path in order to duplicate the conformant message pair. This will directly provide new conformant message pairs for free.

The next step is now to build a main differential path with the tool from De Cannière and Rechberger, containing as much auxiliary paths as possible (of course while favoring $A P_{2}$ instead of $A P_{1}$, the latter being less powerful). We refer to 5 for the details of this algorithm. The tool works well for SHA-0 as for SHA-1 and given a random chaining variable, it is easy to find a main differential path containing at least five auxiliary paths, with at least three $A P_{2}$ characteristics. 


\begin{tabular}{|c||c|c|}
\hline & $W_{0}$ to $W_{15}$ & $W_{16}$ to $W_{31}$ \\
\hline \hline perturbation mask & 1010000000100000 & \\
\hline \hline differences on $W^{j}$ & 1010000000100000 & 0000000010110110 \\
\hline differences on $W^{j+5}$ & 0101000000010000 & 0000000001011011 \\
\hline differences on $W^{j-2}$ & 0001111100000011 & 0000000000001110 \\
\hline
\end{tabular}

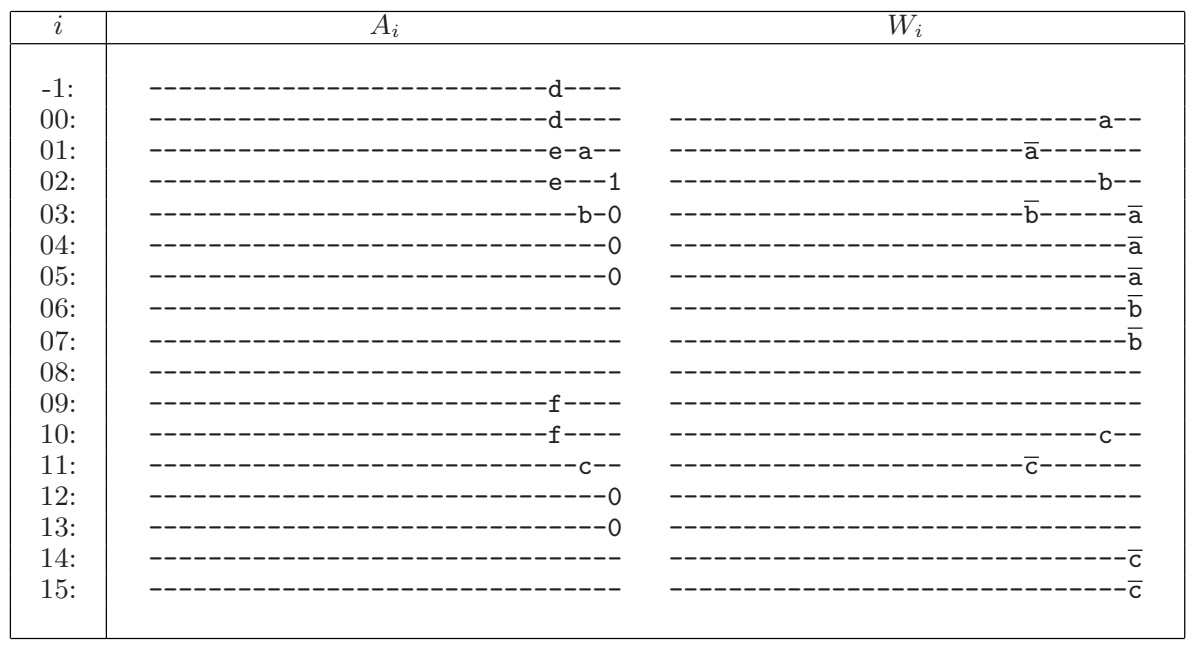

Fig. 4. Auxiliary differential path $A P_{2}$ used during the attack. The first table shows the 32 first steps of the perturbation vector (with the first uncontrolled difference on registers at step 25) and the second gives the constraints forced in order to have probability one local collisions in the early steps in the case where the auxiliary path is positioned at bit $j=2$. The MSB's are on the right and "-" stands for no constraint. The letters represent a bit value and its complement is denoted by an upper bar on the corresponding letter (see 14 for the notations).

Note that this part, as the automated tool, is purely heuristic and often more auxiliary paths can be forced 2 . However, the behavior of the automated tool is quite dependant of the perturbation vector. Thus, among the possible good ones, we chose a perturbation vector (depicted in Table 2) that seemed to work well with the automated search tool. Note that since the perturbation vector remains the same during the two parts of the attack, this property will be true for the two blocks of message.

\footnotetext{
${ }^{2}$ The auxiliary path $A P_{2}$ has one condition on the IV (the bit $d$ in Figure 4) and this harden the task of the attacker to place a lot of them in the main differential trail. However, this is already taken in account in the evaluation. There remains space for improvements on this part but we chose to describe an easy-to-implement attack instead of the best possible but hard to implement one.
} 


\section{The Final Collision Attack}

At this point, we have all the required elements to mount the attack and analyze it. Its total complexity will be the addition of the complexities of the collision search for both block 3 . Note that, unlike for the 2-block collision attacks for SHA-1 where the first block complexity is negligible compared to the second block one, here our perturbation vector imposes the same raw complexity for both blocks.

\subsection{A Method of Comparison}

As observed in [6]14, there are many different collision search speeding techniques for the SHA family of hash functions. However, their real cost is often blurry and it becomes hard to compare them. Thus, it has been advised to measure the efficiency of those tools with an efficient implementation of the various hash functions attacked, for example by using OpenSSL 22. For any computer utilized, one can give the complexity of the attack in terms of number of function calls of the hash function with an efficient implementation, which is relatively independent of the computer used.

In their paper [18, Naito et al. claimed a complexity of $2^{36}$ SHA-0 calls for their collision attack. However, their implementation required approximatively 100 hours on average in order to find one collision on a PentiumIV 3, 4 GHz. 100 hours of SHA-0 computations on this processor would correspond to $2^{40,3}$ SHA-0 calls approximatively with OpenSSL, which is far from the claimed complexity.

Thus, in this paper, we chose to handle the complexities in terms of number of SHA- 0 calls with OpenSSL in order to allow an easy comparison. The time measurements have been done on a single $\mathrm{PC}$ with an AMD Opteron 2, $2 \mathrm{GHz}$ processor.

\subsection{Without Collision Search Speedup}

Without even using boomerang attacks, our new differential paths already provides an improvement on the best known collision attack against SHA-0. Indeed, in our perturbation vector, 42 conditions remain after step 17 . However, by refining the differential path utilized (i.e. by forcing some conditions just before step 17, without any impact on the complexity since located in the early steps), one can easily take care of the two conditions from step 17 before beginning the collision search. Therefore, we are finally left with 40 conditions per message block. Since for each basic message pair tested during the collision search only one quarter of a whole SHA-O is computed in average, we expect a complexity of $2^{40} / 2^{2}=2^{38}$ SHA-0 evaluations for one block and thus a total complexity of $2^{39}$ SHA-0 evaluations for one complete collision. This theoretical complexity is fully confirmed by practical implementation $\left(2^{37,9}\right.$ and $2^{37,8}$ SHA-0 evaluations

\footnotetext{
${ }^{3}$ One can argue that the cost of the NL-characteristics automated search tool has also to be counted. However, unlike in the SHA-1 case, for SHA-0 the number of possible collisions that can be generated with only one full differential path construction is really big. Thus, this cost becomes largely negligible compared to the collision search.
} 
Table 3. Message instance for a 2-block collision: $H\left(M_{1}, M_{2}\right)=H\left(M_{1}^{\prime}, M_{2}^{\prime}\right)=$ $A_{2}|| B_{2}|| C_{2}|| D_{2}|| E_{2}$, computed according to the differential path given in Tables 5 6. 7 and 8 from appendix

\begin{tabular}{|c|c|c|c|c|}
\hline & \multicolumn{2}{|c|}{$1^{\text {st }}$ block } & \multicolumn{2}{|c|}{$2^{\text {nd }}$ block } \\
\hline & $M_{1}$ & $M_{1}^{\prime}$ & $M_{2}$ & $M_{2}^{\prime}$ \\
\hline$W_{0}$ & $0 \times 4643450 \mathrm{~b}$ & $0 \times 46434549$ & 0x9a74cf70 & 0x9a74cf32 \\
\hline$W_{1}$ & 0x41d35081 & $0 \mathrm{x} 41 \mathrm{~d} 350 \mathrm{c} 1$ & 0x04f9957d & $0 x 04 f 9953 d$ \\
\hline$W_{2}$ & 0xfe16dd9b & 0xfe16dddb & 0xee26223d & 0xee26227d \\
\hline$W_{3}$ & 0x3ba36244 & 0x3ba36204 & $0 \times 9 a 06 e 4 b 5$ & $0 x 9 a 06 e 4 f 5$ \\
\hline$W_{4}$ & 0xe6424055 & $0 \times 66424017$ & 0xb8408af6 & 0x38408ab4 \\
\hline$W_{5}$ & $0 \mathrm{x} 16 \mathrm{ca} 44 \mathrm{a} 0$ & $0 \times 96 \mathrm{ca} 44 \mathrm{a} 0$ & 0xb8608612 & $0 \times 38608612$ \\
\hline$W_{6}$ & $0 \times 20 f 62444$ & 0xa0f62404 & $0 x 8 b 7 e 0 f e a$ & 0x0b7e0faa \\
\hline$W_{7}$ & $0 \times 10 f 7465 a$ & $0 x 10 f 7465 a$ & $0 x e 17 e 363 c$ & 0xe17e363c \\
\hline$W_{8}$ & 0x5a711887 & $0 x 5 a 7118 c 5$ & 0xa2f1b8e5 & 0xa2f1b8a7 \\
\hline$W_{9}$ & $0 \times 51479678$ & 0xd147963a & 0xca079936 & $0 \times 4 a 079974$ \\
\hline$W_{10}$ & $0 \times 726 a 0718$ & $0 \times 726 a 0718$ & $0 \times 02 f 2 a 7 c b$ & $0 \times 02 f 2 a 7 c b$ \\
\hline$W_{11}$ & $0 \times 703 f 5 b f b$ & $0 \times 703 f 5 b b 9$ & $0 x f 724 e 838$ & $0 x f 724 e 87 a$ \\
\hline$W_{12}$ & 0xb7d61841 & 0xb7d61801 & $0 \times 37 f f c 03 a$ & $0 \times 37 f f c 07 a$ \\
\hline$W_{13}$ & $0 x a 5280003$ & $0 \times a 5280041$ & $0 \times 53 a a 8 c 43$ & 0x53aa8c01 \\
\hline$W_{14}$ & 0x6b08d26e & $0 \times 6 b 08 d 26 c$ & 0x90811819 & 0x9081181b \\
\hline$W_{15}$ & $0 \mathrm{x} 2 \mathrm{e} 4 \mathrm{df} 0 \mathrm{~d} 8$ & 0xae4df0d8 & $0 \times 312 d 423 e$ & $0 x b 12 d 423 e$ \\
\hline
\end{tabular}

\begin{tabular}{|c|c|c|c|c|}
\hline$A_{2}$ & $B_{2}$ & $C_{2}$ & $D_{2}$ & $E_{2}$ \\
\hline 0x6f84b892 & 0x1f9f2aae & 0x0dbab75c & 0x0afe56f5 & 0xa7974c90 \\
\hline
\end{tabular}

on average for the first and second blocks respectively). Our computer needs 39 hours on average in order to find a collision, which is much faster than Naito et al.'s attack and this with a less powerful processor.

\subsection{Using the Boomerang Improvement}

As one can use many collision search speedup techniques, a good choice can be the boomerang attack for its simplicity of use. We give in the appendix a possible differential path for the first block (Tables [5] and 6) and the second block (Tables 7 and 8). The notations used are also given in the appendix in Table 4 The chaining variable of the second block is the output of the first valid message pair found (i.e. conformant to the whole differential path) for the first block. As for the previous subsection, we are left with 40 conditions for each blocks since the two conditions from step 17 can be easily cancelled before the collision search. The differential path for the second block possesses 5 auxiliary paths. However, as explained before, it is possible to build NL-characteristics containing more auxiliary paths but we only take in account the average case and not the best case 
of behavior of the automated NL-characteristics search tool. For example, one can check that there are $3 A P_{2}$ auxiliary paths in bit positions $j=\{17,22,30\}$ and $2 A P_{1}$ auxiliary paths in bit positions $j=\{9,11\}$ for the second block. The first block case is particular since the chaining variable is the predefined $I V$ which is highly structured. This particular structure greatly improves our capability to place auxiliary paths since the condition on the chaining variable for $A P_{2}$ is verified on much more bit positions than what someone would expect in the random case. Thus, for the first block one can place 2 more $A P_{2}$ on average and one can check in Table 5 that there are $5 A P_{2}$ auxiliary paths in bit positions $j=\{10,14,19,22,27\}$ and $2 A P_{1}$ auxiliary paths in bit positions $j=\{9,11\}$.

In theory, with $k$ auxiliary paths, one would expect an improvement of a factor $2^{k}$. However, this slightly depends on the type and the number of auxiliary paths used. Obviously, compared to the $A P_{1}$ auxiliary path, using the $A P_{2}$ type is better during the collision search. Thus, we expect an improvement of the attack of a factor approximatively $2^{7}=128$ for the first block and $2^{5}=32$ for the second one. We get something close in practice with a measured complexity of $2^{32,2}$ and $2^{33}$ function calls for the first and second block respectively. This leads to a final complexity for a collision on the whole SHA-0 of $2^{33,6}$ function calls, which compares favourably in theory and practice to the best known collision attack on this hash function: our computer can generate a 2-block collision for SHA-0 in approximatively one hour on average (instead of 100 hours of computation on a faster processor for the best known attack). For proof of concept, we provide in Table 3 a 2-block message pairs that collides with SHA-0.

\section{Conclusion}

In this paper, we introduced a new attack against SHA-0. By relaxing the previously established constraints on the perturbation vector, we managed to find better candidates. Then, as a collision search speedup technique, we applied on those candidates the boomerang attack which provides a good improvement with a real practicality of use. This work leads to the best collision attack on SHA-0 so far, requiring only one hour of computation on an average PC. Yet, there stills space for further improvements as some parts of the attack are heuristic. Moreover, this work shows the efficiency of the dual use of the boomerang attack for hash functions combined with a differential path automated search tool.

\section{References}

1. Bellare, M., Ristenpart, T.: Multi-Property-Preserving Hash Domain Extension and the EMD Transform. In: Lai, X., Chen, K. (eds.) ASIACRYPT 2006. LNCS, vol. 4284, pp. 299-314. Springer, Heidelberg (2006)

2. Biham, E., Chen, R.: Near-Collisions of SHA-0. In: Franklin, M.K. (ed.) CRYPTO 2004. LNCS, vol. 3152, pp. 290-305. Springer, Heidelberg (2004)

3. Biham, E., Chen, R., Joux, A., Carribault, P., Lemuet, C., Jalby, W.: Collisions of SHA-0 and Reduced SHA-1. In: Cramer, R. (ed.) EUROCRYPT 2005. LNCS, vol. 3494, pp. 36-57. Springer, Heidelberg (2005) 
4. Biham, E., Dunkelman, O.: A Framework for Iterative Hash Functions: HAIFA. In: Proceedings of Second NIST Cryptographic Hash Workshop (2006), Www.csrc.nist.gov/pki/HashWorkshop/2006/program_2006.htm

5. De Cannière, C., Rechberger, C.: Finding SHA-1 Characteristics: General Results and Applications. In: Lai, X., Chen, K. (eds.) ASIACRYPT 2006. LNCS, vol. 4284, pp. 1-20. Springer, Heidelberg (2006)

6. De Cannire, C., Mendel, F., Rechberger, C.: Collisions for 70-step SHA-1: On the Full Cost of Collision Search. In: Adams, C., Miri, A., Wiener, M. (eds.) SAC 2007. LNCS, vol. 4876. Springer, Heidelberg (2007)

7. Chabaud, F., Joux, A.: Differential Collisions in SHA-0. In: Krawczyk, H. (ed.) CRYPTO 1998. LNCS, vol. 1462, pp. 56-71. Springer, Heidelberg (1998)

8. Damgård, I.: A Design Principle for Hash Functions. In: Brassard, G. (ed.) CRYPTO 1989. LNCS, vol. 435, pp. 416-427. Springer, Heidelberg (1990)

9. Dean, R.D.: Formal Aspects of Mobile Code Security. PhD thesis, Princeton University (1999)

10. Dobbertin, H.: Cryptanalysis of MD4. In: Gollmann, D. (ed.) FSE 1996. LNCS, vol. 1039, pp. 53-69. Springer, Heidelberg (1996)

11. Menezes, A.J., Vanstone, S.A., Van Oorschot, P.C.: Handbook of Applied Cryptography. CRC Press, Inc., Boca Raton (1996)

12. Hoch, J.J., Shamir, A.: Breaking the ICE - Finding Multicollisions in Iterated Concatenated and Expanded (ICE) Hash Functions. In: Robshaw, M.J.B. (ed.) FSE 2006. LNCS, vol. 4047, pp. 179-194. Springer, Heidelberg (2006)

13. Joux, A.: Multi-collisions in Iterated Hash Functions. In: Franklin, M. (ed.) CRYPTO 2004. LNCS, vol. 3152, pp. 306-316. Springer, Heidelberg (2004)

14. Joux, A., Peyrin, T.: Hash Functions and the (Amplified) Boomerang Attack. In: Menezes, A. (ed.) CRYPTO 2007. LNCS, vol. 4622, pp. 244-263. Springer, Heidelberg (2007)

15. Kelsey, J., Schneier, B.: Second Preimages on $n$-bit Hash Functions for Much Less Than $2^{n}$ Work. In: Cramer, R. (ed.) EUROCRYPT 2005. LNCS, vol. 3494, pp. 474-490. Springer, Heidelberg (2005)

16. Manuel, S.: Cryptanalyses Différentielles de SHA-0. Mémoire pour l'obtention du Mastère Recherche Mathematiques Applications au Codage et à la Cryptographie. Université Paris 8 (2006), http://www-rocq.inria.fr/codes/Stephane.Manuel

17. Merkle, R.C.: One Way Hash Functions and DES. In: Brassard, G. (ed.) CRYPTO 1989. LNCS, vol. 435, pp. 428-446. Springer, Heidelberg (1990)

18. Naito, Y., Sasaki, Y., Shimoyama, T., Yajima, J., Kunihiro, N., Ohta, K. (eds.): ASIACRYPT 2006. LNCS, vol. 4284, pp. 21-36. Springer, Heidelberg (2006)

19. National Institute of Standards and Technology. FIPS 180: Secure Hash Standard (May 1993), http://csrc.nist.gov

20. National Institute of Standards and Technology. FIPS 180-1: Secure Hash Standard (April 1995), http://csrc.nist.gov

21. National Institute of Standards and Technology. FIPS 180-2: Secure Hash Standard (August 2002), http://csrc.nist.gov

22. OpenSSL. The Open Source toolkit for SSL/TLS (2007), http://www.openssl.org/source

23. Rivest, R.L.: RFC 1321: The MD5 Message-Digest Algorithm (April 1992), http://www.ietf.org/rfc/rfc1321.txt

24. Rivest, R.L.: RFC 1320: The MD4 Message Digest Algorithm (April 1992), http://www.ietf.org/rfc/rfc1320.txt

25. Wagner, D.: The Boomerang Attack. In: Knudsen, L.R. (ed.) FSE 1999. LNCS, vol. 1636, pp. 156-170. Springer, Heidelberg (1999) 
26. Wang, X., Lai, X., Feng, D., Chen, H., Yu, X.: Cryptanalysis of the Hash Functions MD4 and RIPEMD. In: Cramer, R. (ed.) EUROCRYPT 2005. LNCS, vol. 3494, pp. 1-18. Springer, Heidelberg (2005)

27. Wang, X., Yu, H.: How to Break MD5 and Other Hash Functions. In: Cramer, R. (ed.) EUROCRYPT 2005. LNCS, vol. 3494, pp. 19-35. Springer, Heidelberg (2005)

28. Wang, X., Yu, H., Yin, Y.L.: Efficient Collision Search Attacks on SHA-0. In: Shoup, V. (ed.) CRYPTO 2005. LNCS, vol. 3621, pp. 1-16. Springer, Heidelberg (2005)

29. Wang, X., Yin, Y.L., Yu, H.: Finding Collisions in the Full SHA-1. In: Shoup, V. (ed.) CRYPTO 2005. LNCS, vol. 3621, pp. 17-36. Springer, Heidelberg (2005)

\section{Appendix}

Table 4. Notations used in [5] for a differential path: $x$ represents a bit of the first message and $x^{*}$ stands for the same bit of the second message

\begin{tabular}{|c|c|c|c|c|}
\hline$\left(x, x^{*}\right)$ & \multicolumn{4}{|c|}{$(0,0)(1,0)(0,1)(1,1)$} \\
\hline$?$ & $\checkmark$ & $\checkmark$ & $\checkmark$ & $\checkmark$ \\
\hline- & $\checkmark$ & - & - & $\checkmark$ \\
\hline $\mathrm{x}$ & - & $\checkmark$ & $\checkmark$ & - \\
\hline 0 & $\checkmark$ & - & - & - \\
\hline $\mathrm{u}$ & - & $\checkmark$ & - & - \\
\hline $\mathrm{n}$ & - & - & $\checkmark$ & - \\
\hline 1 & - & - & - & $\checkmark$ \\
\hline$\#$ & - & - & - & - \\
\hline
\end{tabular}

\begin{tabular}{|c|c|c|c|c|}
\hline$\left(x, x^{*}\right)$ & \multicolumn{4}{|c|}{$(0,0)(1,0)(0,1)(1,1)$} \\
\hline 3 & $\checkmark$ & $\checkmark$ & - & - \\
\hline 5 & $\checkmark$ & - & $\checkmark$ & - \\
\hline 7 & $\checkmark$ & $\checkmark$ & $\checkmark$ & - \\
\hline A & - & $\checkmark$ & - & $\checkmark$ \\
\hline B & $\checkmark$ & $\checkmark$ & - & $\checkmark$ \\
\hline $\mathrm{C}$ & - & - & $\checkmark$ & $\checkmark$ \\
\hline D & $\checkmark$ & - & $\checkmark$ & $\checkmark$ \\
\hline $\mathrm{E}$ & - & $\checkmark$ & $\checkmark$ & $\checkmark$ \\
\hline
\end{tabular}


Table 5. Steps 1 to 39 of the main differential path of the first block

\begin{tabular}{|c|c|c|}
\hline$i$ & $A_{i}$ & $W_{i}$ \\
\hline$-4:$ & 00001111010010111000011111000011 & \\
\hline$-3:$ & 01000000110010010101000111011000 & \\
\hline-2 : & 01100010111010110111001111111010 & \\
\hline$-1:$ & 11101111110011011010101110001001 & \\
\hline 00 : & 01100111010001010010001100000001 & $0100111001001011000001010 \mathrm{n} 0010 \mathrm{u} 1$ \\
\hline 01: & $1110110111111111100111011 \mathrm{n} 1111 \mathrm{u} 0$ & $0100000011011011010100001 \mathrm{n} 000000$ \\
\hline 02 : & $01100111011111111101 \mathrm{n} 10101101010$ & $1111011000011110100111011 \mathrm{n} 011011$ \\
\hline 03: & 001101010010100 n00001100nOuuuuuo & $0011100010101001011100100 u 000101$ \\
\hline 04: & $111100000 \mathrm{nu} 000001010110001110000$ & u110010001000000010100000u0101n1 \\
\hline 05 : & $00111 \mathrm{n} 00000010011000100000 \mathrm{u} 0 \mathrm{n} 1 \mathrm{u} 1$ & n0010100110010000101010010100000 \\
\hline 06 : & $10110101110110110000101 u 100 u 1001$ & n010001011110100001111000u000100 \\
\hline 07: & 100unnnnnnnnn0100nu0100101u11001 & 00010010111101000101011001011010 \\
\hline 08: & $1000011100001 \mathrm{n} 000 \mathrm{n} 100 \mathrm{u} 0 \mathrm{n} 010 \mathrm{nn} 001$ & $0101101001110001000110001 \mathrm{n} 0001 \mathrm{u} 1$ \\
\hline 09: & 0010000000000010 un00nu1u1un01100 & n101000101000111100101100u1110no \\
\hline 10: & 11100110100101000 nu01u10un00n100 & 01111010011000100100011100011000 \\
\hline 11: & 011110001110001101 nuu10101000101 & $0111000100110111010110011 u 1110 u 0$ \\
\hline 12: & $01001101011010000010 u 0000 \mathrm{n} 110000$ & $10110111110101-----1-----u 000001$ \\
\hline 13: & $010110011100000----010-0-01001 \mathrm{u} 0$ & 101001010---------------n0000u1 \\
\hline 14: & 10111100--------------1--110u011 & $01101-0----0--1----0---0-1-011 \mathrm{u} 0$ \\
\hline 15: & $10100--0-0-1-\mathrm{u} 0100$ & n0101-0----0--1----0---0-1-11000 \\
\hline 16: & --01-------------------------n0011 & 010001110---------------00101no \\
\hline 17: & ------------------------------1n- & n1000-0----1--1----1---0-u-10011 \\
\hline 18: & 1------------------------------0-- & $01000-0----1--1----0---0-0-011 \mathrm{u} 0$ \\
\hline 19: & 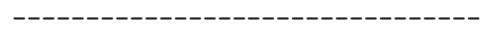 & n00110100---------------0001011 \\
\hline 20: & 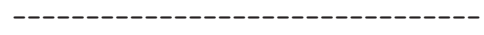 & n0110-0----1-------0-----0-000u1 \\
\hline 21: & 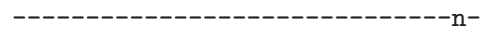 & u1100-1------------------u-10111 \\
\hline 22: & 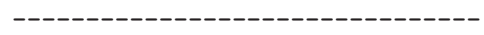 & 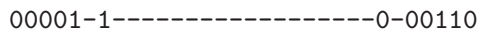 \\
\hline 23: & 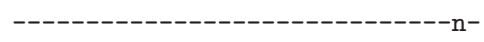 & n1011-1-----0--------0------u-11001 \\
\hline 24: & 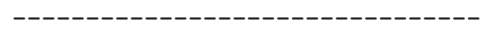 & u0000-0---------------1-11100 \\
\hline 25: & 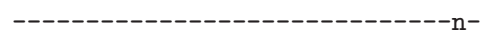 & 01101-1-------------------u-10111 \\
\hline 26: & 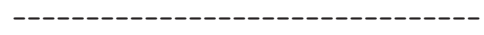 & u1010-1-----0-------1------0-011u0 \\
\hline 27: & -------- & 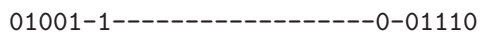 \\
\hline 28: & ------- & ------------1-11011 \\
\hline 29: & ---- & $------0-00010$ \\
\hline 30: & ---- & 01101-1---------------1-10010 \\
\hline $31:$ & --- & $-----0-0-01001$ \\
\hline $32:$ & --- & $---1-00100$ \\
\hline 33: & --- & ------------1-11101 \\
\hline 34: & ------- & 00010-0----------------0-010u0 \\
\hline 35: & 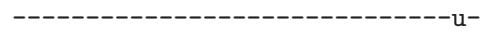 & 10001-0----------------n-10110 \\
\hline 36: & 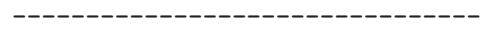 & 11100-0-----------------0-000u1 \\
\hline 37: & 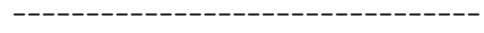 & n0010-0-------------0-001u0 \\
\hline 38: & 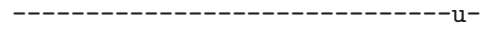 & n1101-0------------n-11110 \\
\hline 39: & --------------- & n1100-1---- \\
\hline
\end{tabular}


Table 6. Steps 40 to 80 of the main differential path of the first block

\begin{tabular}{|c|c|c|}
\hline$i$ & $A_{i}$ & $W_{i}$ \\
\hline & & \\
\hline 40: & ----- & n1111-0--------------0-10000 \\
\hline 41: & 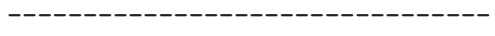 & n0010-1----------------0-11010 \\
\hline 42: & ------ & n0100-0-------------1-110u1 \\
\hline 43: & $----u-$ & $00000-1---$ \\
\hline 44: & --- & $00011-0----$ \\
\hline 45: & ---- & n0111-1---------------1-10110 \\
\hline 46: & ----------- & n0111-1------------------0-00010 \\
\hline 47: & --------------- & u0010-1-------------------1-00000 \\
\hline 48: & 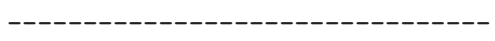 & 01101-0-----------------0-010no \\
\hline 49: & 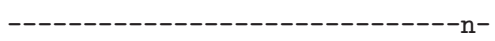 & 11111-1--------------------u-10011 \\
\hline 50: & ---------------------------------- & 01000-1----------------0-100u 0 \\
\hline 51: & ---------------------------------- & u1110-1--------------------0-10010 \\
\hline $52:$ & ---------------------------------- & n1101-1-------------------1-11110 \\
\hline 53: & 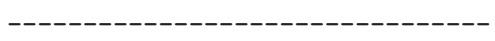 & n0001-1---------------1-001u0 \\
\hline 54: & 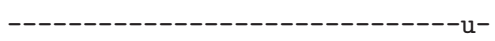 & 11011-0-----------------n-11110 \\
\hline 55: & ---------------------------------- & 10001-0--------------0-000no \\
\hline $56:$ & 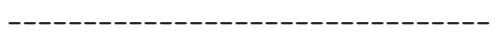 & n0111-1------------------0-001n1 \\
\hline 57: & -------------n- & $\mathrm{n} 0110-1-------------u-11101$ \\
\hline 58: & ---------------- & u1110-1-------------------1-11001 \\
\hline 59: & $-\cdots---n-$ & $-----u-010 u 1$ \\
\hline 60: & $------u-$ & $-----n-100 n 1$ \\
\hline 61: & --- & $-----0-010 \mathrm{n} 1$ \\
\hline 62: & ---- & 01111-1-------------------1-11111 \\
\hline 63: & ----------- & 10011-1------------------0-00010 \\
\hline 64: & --------------- & n1000-0------------------0-10110 \\
\hline 65: & ---------------- & 01000-0----------------1-00011 \\
\hline $66:$ & 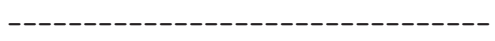 & 01000-0----------------0-101u1 \\
\hline 67: & -------------------------------- u- & 01001-0-------------------n-01001 \\
\hline 68: & ---------------------------------- & 10001-0---------------0-100u \\
\hline 69: & 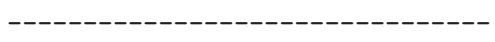 & u0010-1------------------1-11000 \\
\hline 70: & 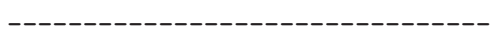 & u1010-0---------------1-011n1 \\
\hline 71: & 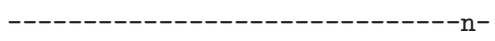 & u0101-0-------------------u-01101 \\
\hline $72:$ & 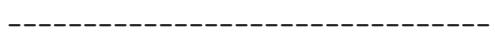 & 00011-1----------------0-100u0 \\
\hline 73: & --------------------------------- & n1010-1------------11000 \\
\hline 74: & --------------------------------- & n1100-0--------- $0-10010$ \\
\hline 75: & ------------- & u1110-1------------------1-110n1 \\
\hline 76: & --------------------------------n- & 11011-1--------------------u-00100 \\
\hline 77: & -------------- & 00111-0-----------------1-000n1 \\
\hline 78: & ------------ & n0011-0---------------1-11101 \\
\hline 79: & ------------------------------------ & u0101-0------------------1-01000 \\
\hline 80: & --- & \\
\hline
\end{tabular}


Table 7. Steps 1 to 39 of the main differential path of the second block

\begin{tabular}{|c|c|c|}
\hline$i$ & $A_{i}$ & $W_{i}$ \\
\hline-4 : & $111011010000101010001110101010 u 1$ & \\
\hline$-3:$ & 01000110011100010110101100101000 & \\
\hline-2 : & 10010000011011111010001110100111 & \\
\hline$-1:$ & 11100110001011011100010100001001 & \\
\hline $00:$ & 01011110101010111100001100111101 & $1001101001110110110011110 u 1100 \mathrm{n} 0$ \\
\hline 01: & u0111011010011100010111unn1010n1 & $0000010010111001100101010 u 111101$ \\
\hline 02: & $11010011001110011011 u 000110 u 0111$ & $1110111000100100001000100 \mathrm{n} 111101$ \\
\hline 03: & $111001111000010 u 000 u n n n n n n 000100$ & $1001101001000110011001001 \mathrm{n} 110101$ \\
\hline 04: & u0100101unn01010000u100011110110 & u011100001000000000010101u1101u0 \\
\hline 05: & n000un001000011u00100000000nn0n0 & u0111000011000000000011000010010 \\
\hline 06: & nnn0010001011110011100n1nu1u011u & $\mathrm{u} 000101101111110100001011 \mathrm{u} 101010$ \\
\hline 07: & 10nuuuuuuuuuuuuu11100n00un0u1001 & 11100001011111111111011000111100 \\
\hline 08: & 0001111100000000 unnn11010001n001 & $1010001011110001101110001 \mathrm{u} 1001 \mathrm{n} 1$ \\
\hline 09: & 00000111111111111110001 n111un111 & u100101000000111100110010n1101u0 \\
\hline 10: & 1110110110111111110100nu111uu011 & 00000010111100001010011111001011 \\
\hline 11: & $00111110010001010011011 \mathrm{uu} 0 \mathrm{n} 000 \mathrm{u} 0$ & $1111011101100100111010101 \mathrm{n} 1110 \mathrm{n} 0$ \\
\hline 12: & 010001101000111000111111nuu1u011 & $001101111111-------------n 111010$ \\
\hline 13: & $101010000000----0--------01111 \mathrm{u} 0$ & 01010---------------u0000u1 \\
\hline 14: & $00110001-----------------1010010$ & 10010------0----1--------00110n1 \\
\hline 15: & 10011--------------10101n0 & n0110-------0----1--------0111110 \\
\hline 16: & -----011000 & 10000----------11010u1 \\
\hline 17: & $------n-$ & u1000------1----1--------u100111 \\
\hline 18: & 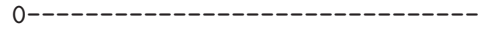 & $01100------1----0-------01111 \mathrm{u} 0$ \\
\hline 19: & 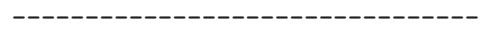 & n1010-------------------1110100 \\
\hline 20: & ------------------- & u1000------1-----------10000n1 \\
\hline 21: & 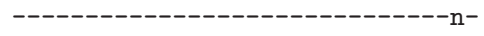 & n1101-------------------u010011 \\
\hline 22: & 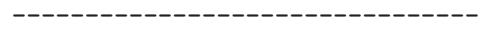 & 11101-------------------1100010 \\
\hline 23: & 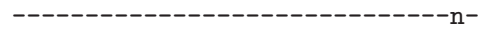 & u1011-------0-------------u110101 \\
\hline 24: & 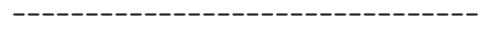 & n1001------------0010110 \\
\hline 25: & 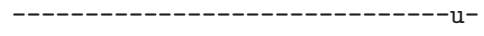 & 00010----------------n001011 \\
\hline 26: & 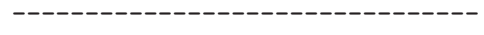 & u0001------1-------------01110u0 \\
\hline 27: & 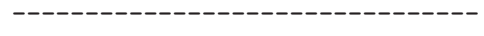 & 10111------------------0011001 \\
\hline 28: & -------- & n1110----------1101001 \\
\hline 29: & --------- & u0000-------------------0010100 \\
\hline 30: & ------ & 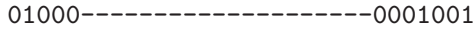 \\
\hline 31: & --- & 01011------------------1000101 \\
\hline 32: & --- & 00101-------------------1010111 \\
\hline 33: & --- & 11000------------------0010001 \\
\hline 34: & --- & 01110----------------00000no \\
\hline 35: & $---n-$ & 10101------------------u101001 \\
\hline 36: & ---- & 10011------------10110u1 \\
\hline 37: & -------- & n1000-------------01100u0 \\
\hline 38: & 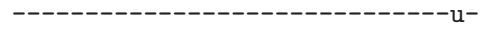 & n1001-------------n010100 \\
\hline 39: & 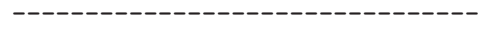 & n0001----- \\
\hline
\end{tabular}


Table 8. Steps 40 to 80 of the main differential path of the second block

\begin{tabular}{|c|c|c|}
\hline$i$ & $A_{i}$ & $W_{i}$ \\
\hline & & \\
\hline 40: & ---- & 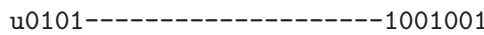 \\
\hline 41: & ------------------ & n0100------------------0010111 \\
\hline 42: & 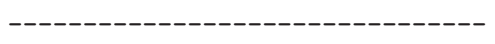 & u0000--------------------01100u1 \\
\hline 43: & 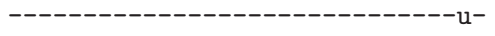 & 00111------------------n101101 \\
\hline 44: & 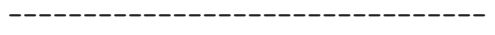 & $10001----------------01011 \mathrm{no}$ \\
\hline 45: & ---------- & n0011-----------------1010000 \\
\hline 46: & --- & ----1100111 \\
\hline 47: & ---- & n0011------------------0011000 \\
\hline 48: & ---- & $11101-------$ \\
\hline 49: & $---------u-$ & $-----n 001000$ \\
\hline 50: & ----------- & 01110--------------------11100no \\
\hline 51: & --------------- & n0111---------------------0111000 \\
\hline $52:$ & -------------- & n0001--------------------1101011 \\
\hline 53: & ------------ & n0100-------- \\
\hline $54:$ & -------------u- & $11000-------$ \\
\hline $55:$ & ---------- & $-------00001 \mathrm{n} 0$ \\
\hline 56 : & --------------- & u1100---------------------10001uc \\
\hline $57:$ & 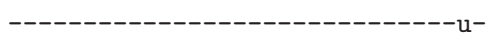 & u0001---------------------n110000 \\
\hline 58: & 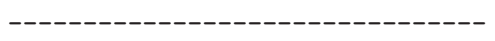 & n1000---------------------1101011 \\
\hline 59: & 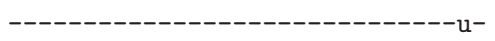 & u1111----------------------n0000u1 \\
\hline 60: & 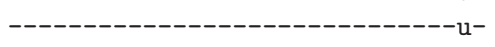 & n0010---------- n0100no \\
\hline 61: & ---------------------------------- & 01100--------------------10100n1 \\
\hline $62:$ & ---------------------------------- & $11001--------------------0101000$ \\
\hline 63: & ---------------------------------- & 01100--------------------0000100 \\
\hline 64: & --------------- & n0011--------------------0101001 \\
\hline 65: & 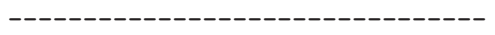 & 00101---------------------0101000 \\
\hline 66: & ---------------- & 01011---------------------11101no \\
\hline 67: & --------------------------------n- & $11111---------------------u 100000$ \\
\hline 68: & ---------------------------------- & $11110-------------------10100 \mathrm{n} 1$ \\
\hline 69: & ------------------------------------ & n0100--------------------1010011 \\
\hline 70: & ----------------------------------- & n0010--------------------00011no \\
\hline $71:$ & --------------------------------n- & n0100--------------------u100001 \\
\hline $72:$ & -------------- & $10011-----------------10101 \mathrm{u} 1$ \\
\hline 73: & --------------- & n1001----------------------0010111 \\
\hline $74:$ & ------------------------------------ & n0101-------------------1101110 \\
\hline 75: & --------------- & u1111----------------------11001n1 \\
\hline 76: & $----------n-$ & 01100-------------------u111110 \\
\hline 77: & -------------- & $00001---------------11010 \mathrm{no}$ \\
\hline 78: & ------------ & n0111------------------1101000 \\
\hline 79: & -------------- & n0001----------------------0110011 \\
\hline 80: & 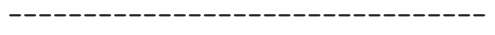 & \\
\hline
\end{tabular}

